\title{
EFFECT OF 6-WEEK INTERVAL TRAINING PROTOCOL ON RUNNING PERFORMANCE OF MESFIN INDUSTRIAL ENGINEERING (MIE)TRACK TRANIEE ATHLETES
}

\author{
Soumitra Mandal ${ }^{\mathrm{a}, *}$ and Samson Wondirad ${ }^{\mathrm{b}}$ \\ ${ }^{a}$ Department of Sport Science, College of Natural and Computational Sciences, Mekelle \\ University P.O.Box: 231, Ethiopia, Africa. \\ ${ }^{\mathrm{b}}$ Department of Sport Science, College of Natural Sciences, Jimma University, \\ P.O.Box.375, Jimma, Ethiopia, Africa. \\ *Corresponding Author Ph: +25190233 5495; Email: smwonde@gmail.com
}

\section{DOI: 10.26524/1411}

\begin{abstract}
Interval Training is the newly used training program because of its effectiveness in developing both the aerobic and lactate energy systems. However, there are controversies due to different kinds of training program in choosing and using them to trains an athlete. Therefore,this study was aimed to see theeffect of 6-week new interval training protocol on running performance of MIE track trainee athletes. Depending on scientific evidence researchers hypothesized that there will be significant difference between pre and post test performance for middle $(800 \mathrm{~m}, 1500 \mathrm{~m})$ and long $(3 \mathrm{Km}, 5 \mathrm{Km} \& 10 \mathrm{Km})$ distance runner. Longitudinal experimental research design was used; pertaining to data collection pre and post and athletes performed interval training 68 sessions over 6 weeks conducted on 15 male and 15 female elite athletes. Statistical analysis such as percentages, mean, standard deviation paired sample t-test, tables and figures by entering the data into SPSS version-20.Thirty elite athletes of age: $18 \pm 2 \mathrm{yrs}$; height $167 \pm 5.8$ centimeter; weight $51.2 \pm 4.29 \mathrm{Kg}$. Significance difference was observed at $\mathrm{P}<0.05$. The athletes showed significant performance improvement in their respective events.
\end{abstract}

Key words: Athletes, Interval training, Middle distance, Long distance, Performance

\section{Introduction}

All track athletes need to do some aerobic training to enhance both their capacity to perform and their ability to recover from training. All runners need to do some repetition training to enhance their running economy, and all runners need to enhance their lactate tolerance and anaerobic thresholds [1]. Track running Performance need intense exercise which involves appropriate proportion of energy contribution from aerobic and anaerobic sources [2].

In already well-trained track athletes, interval training is a well known exercise protocol which is beneficial for weight loss, rehabilitation, and cardiovascular build-up [3]. Training while inspiring hyperoxic gas is a relatively novel strategy which allows athletes to train at a higher intensity and may possibly improve physiological adaptation and subsequent endurance performance [4]. 
It is obvious that when a period of high intensity interval training is supplemented into the already high training volumes of welltrained endurance athletes, further enhancements in both intense and prolonged endurance performance are possible. As well, lower volume high-intensity interval training can maintain endurance performance ability in already well-trained endurance athletes. [5-7].

Correspondingly, interval training have numerous effect among it's benefit Chronic and acute respiratory muscle loading augment that the effect of a 6-week interval program on tolerance of high-intensity intermittent bouts of running [8]. Therefore, the study was to see the effect of new interval training protocol on running performance of MIE track trainee athletes. The researchers hypothesized that there will be significant difference between mean score of pre and post test of middle and long distance runner.

\section{Methodology}

\section{Research Design}

The researcher used longitudinal experimental research design because participants are tested than one test and trained for six weeks. Therefore, the data were collected from Mesfin Industrial Engineering Athletics Sport Club.

\section{Participants}

Healthy athletes $(n=15$ men and 15 women) aged from $18 \pm 2$, elite athletes who took part in national and international competition and $6 \pm 4$ years of experienced volunteer athletes were the subject of this study. Participants were free of risk factors associated with cardiovascular, pulmonary or metabolic disease, deemed safe to begin physical activity, and were not engaged in a regular training program. Other exclusion criteria included medication usage, pregnancy and smoking. The experimental procedures and potential risks were explained prior to the study, and all participants provided written, informed consent.

\section{Training program}

Interval Training recently used because of its effectiveness in developing both the aerobic and lactate threshold energy systems, the recovery in the intervals is a very active 'roll-on', running recovery. The roll-on, active running recovery will depend on the fitness and experience of the athlete. For an experienced athlete a $100 \mathrm{~m}$ roll-on recovery time frequently less than 25-30 seconds. New interval training, then, is a specific type of repetition training where the training effect occurs in the interval between the faster sections. Only repetition training that has the training effect taking place in the interval should be called 'interval training. Hence, the athletes performed interval training 68 sessions over 6 weeks using 100m roll-on running recovery time over a given distance, time and intensity depends on each specific middle and long distance event.

\section{Instruction to subjects}

Before conducting any of the performance tests, participants were required to undergo warm up protocol for 5minute of jogging and a standardized stretching protocol [9] followed by 10minute test. Beside this, subjects were instructed to avoid vigorous exercises for at least $48 \mathrm{hr}$ prior to attending tests. They were 
advised to consume their normal pre-training diet. All the selected anthropometric characteristics and vertical jump were measured from each subject chosen from Mesfin Industrial Engineering.

\section{Procedures}

Body weight

2012 digital manual body glass weighing scale; Capacity: 150kg; Graduation: $0.1 \mathrm{~kg}$; and N.W:1.9KG was used to measure participants' body mass. As the body weight fluctuates during different time of the day, all readings were taken in the evening $(5: 30$ 6:00). The subjects dressed light clothing and barefoot, got on to the each side of the scale with weight distributed evenly between the feet. The measurement was taken three times and the average was taken with the participants standing ahead. The body weight was measured to the nearest of $1 \mathrm{Kg}$.

\section{Height measure}

Stadiometer apparatus is low cost and quick test for measuring height. As equipment, steel ruler and tape measure placed against a wall. Procedure, subject standing height was the measured to the maximum distance from the floor to the highest point on the head, when the subject was facing directly ahead. Shoes should be off, feet together, and arms by the sides. Heels, buttocks and upper back should also be in contact with the wall when the measurement was made. Reliability of stadiometer height measurement can vary throughout the day. So to ensure reliability height should be measured at the same time of day. Height measurement was taken in the morning (6:30-7:30).

\section{Measure of distance}

Middle (800m and $1500 \mathrm{~m}$ ) and long (3000m, 5Km and 10KM) distance event were measured in $(\mathrm{m} / \mathrm{s})$.

\section{Statistical Analyses}

Statistical analysis such as percentages, mean, standard deviation paired sample t-test, tables, pie-chart and figures by entering the data into SPSS version-20.P-values less than 0.05 were considered significant.

\section{Results: Physical characteristics of athletes}

Physical characteristics of participants and gender difference in their respective distance have been clearly shown in the below mentioned Figure1, and Figure 2.
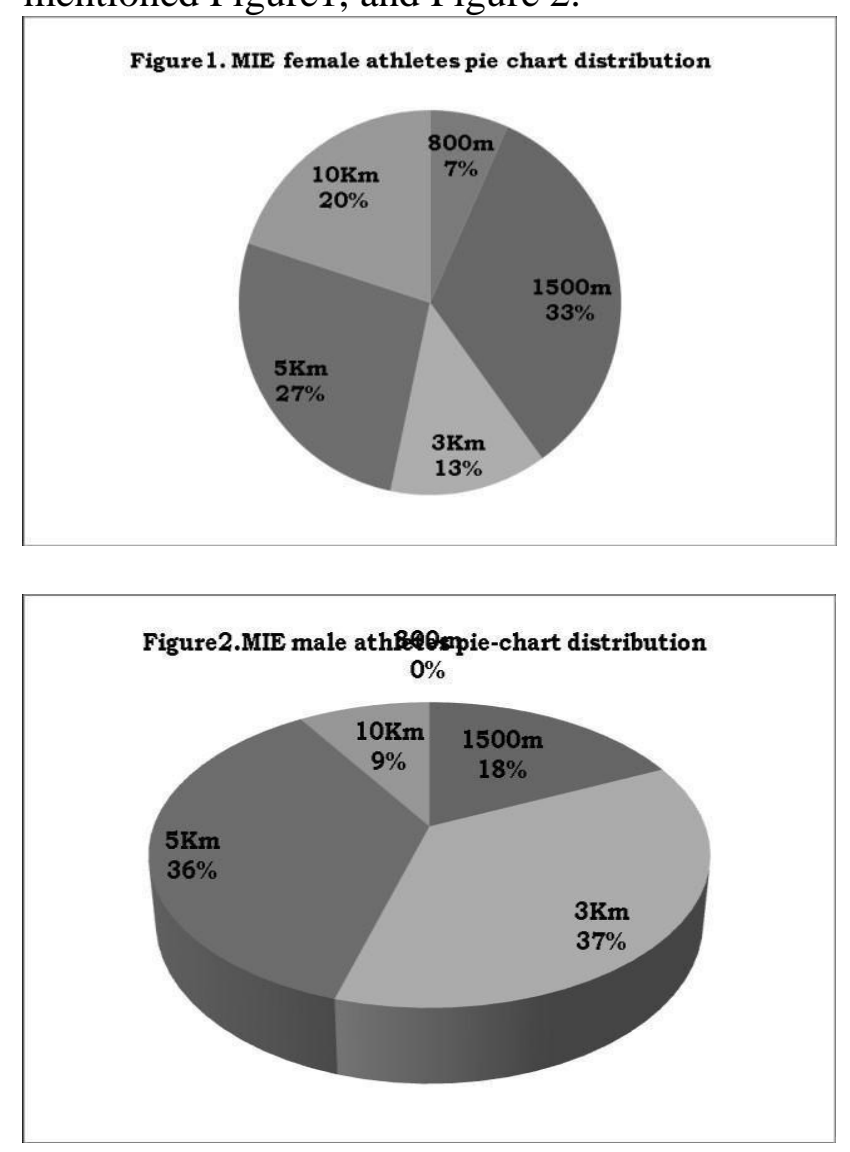
Figure $1 \& 2$. Clearly showed that both male and female athletes were shared $50 \%$ of the total population; all of them are professional in athletics and single they are. Similarly, male athletes are having $1(3.33 \%), 5(16.5 \%), 2(6.66 \%), 4(13.2 \%)$ and $3(9.9 \%)$ in $800 \mathrm{~m}, 1500 \mathrm{~m}, 3000 \mathrm{~m}, 5000 \mathrm{~m}$ and $10,000 \mathrm{~m}$ respectively. while female athletes were took part $4(13.2 \%), 2(6.6 \%), 4(13.2 \%)$, $4(13.2 \%)$ and $1(3.33 \%)$ in $800 \mathrm{~m}$, $1500 \mathrm{~m}, 3000 \mathrm{~m}, 5000 \mathrm{~m}$ and $10,000 \mathrm{~m}$ respectively.

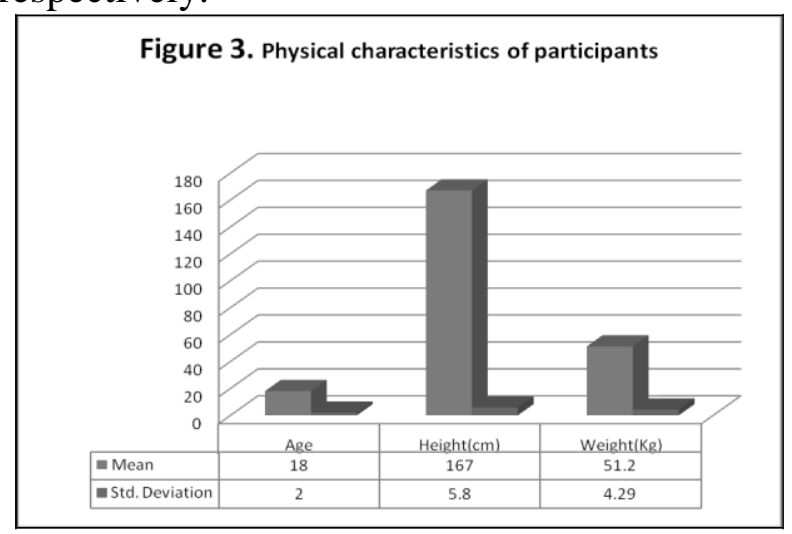

Thirty elite athletes (Mean \pm SD) age: $18 \pm 2$ yrs; height 167 centimeter \pm

5.8 centimeter; weight $51.2 \pm 4.29 \mathrm{Kg}$.

Figure4. Result of Middle \& Long distance runner's performance

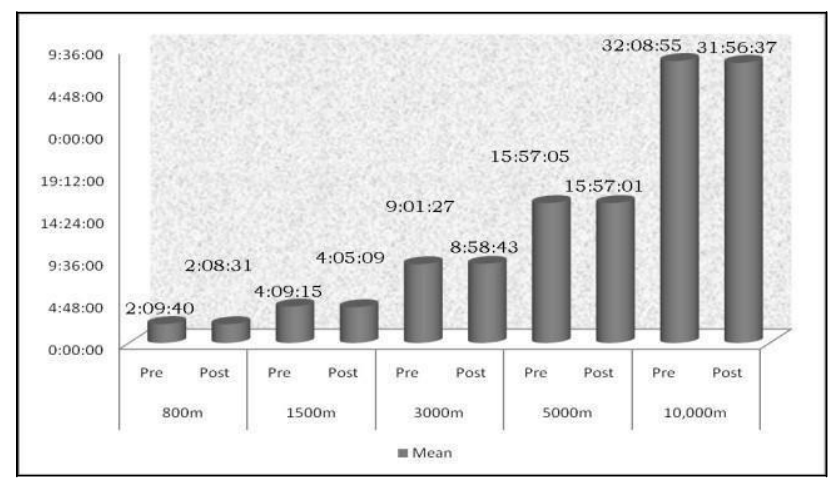

The result of descriptive statistics in pre and post test mean presented in Figure2. As shown in the figure in post test athletes showed performance improvement. (Table 1 Shown in Appendix)

The significant performance difference was calculated using paired sample t-test. As it is demonstrated in Table 1.the null hypothesis was accepted and Significant running performance difference was observed in $800 \mathrm{~m}$, $1500 \mathrm{~m}, 3000 \mathrm{~m}, 5000 \mathrm{~m}$ and $10,000 \mathrm{~m}$.

\section{Discussions}

This study was aimed to report on the basis of longitudinal study, however, we have to keep in mind that conclusions have been drawn on the effect of 6-week new interval training protocol on running performance of Mesfin Industrial Engineering track trainee athletes.

In our case, middle and long distance runners had significantly improved their running performance. Similarly, recent study clearly shows that in well-trained middle distance runners, Smith et al. (1999, 2003) found improvements in $3000 \mathrm{~m}$ running performance when runners performed highintensity interval training twice a week for 4 weeks. Clearly, a short term period of highintensity interval training supplemented into the already high training volumes of welltrained athletes can elicit improvements in both intense and prolonged exercise performance [10]. In the same way, other study supported that interval training in the training programs of distance runners to improve physiological parameters relevant to running performance [11].

In the present study, Interval training method is a well known exercise protocol 
which helps to strengthen and improve one's cardiovascular fitness. Moreover, it helps with weight loss, rehabilitation, general fitness and the reduction of heart disease. Correspondingly, the finding of other research showed that high-intensity interval running program is a beneficial training strategy for enhancing the tolerance to high- intensity intermittent bouts of running [12].

Latest study reported that highintensity interval training has shown that maximal aerobic speed was improved and that 1 . the time of the $40-\mathrm{m}$ sprint was decreased whereas no changes in either parameter were observed during the control period. This study shows that improvements in physical qualities can be made during the in-season period [13]. 2 .

Another study demonstrates the effectiveness of interval training at improving arterial stiffness and autonomic function, however, the metabolic stress was not a 3 . mediator of this effect. In addition, these changes were also independent of improvements in aerobic capacity, which were only induced by training that involved a high 4 . metabolic stress [14]. In contrary one of the recent research revealed, interval training in the competitive season had less effect on 5. endurance and high-intensity performance and physiology in trained endurance cyclists.

\section{Conclusion}

The proposed Hypothesis stands true and this study can conclude that new interval training protocol significantly improve the performance of middle and long distance runner athlete.

\section{Acknowledgment}

We are very grateful to the MIE Coach Mr. Kasahun G/tsadkan. Our Special thankfulness goes to Mr. Somshankar Mukarjee and Department of Sport science at College of Natural and Computational Sciences, Mekelle University, for their unreserved assistance and cooperation for the completion of this research project.

\section{References}

O. P. John, L. P. Naumann, C. J. Soto (2008) Paradigm Shift to the Integrative Big-Five Trait Taxonomy: History, Measurement, and Conceptual Issues, Handbook of personality: Theory and research, NY: Guilford Press.

. P. G. Montgomery, D. B. Pyne, C. L. Minahan, The Physical and Physiological Demands of Basketball Training and Competition, International Journal of Sports Physiology and Performance, 5 (2010) 75-86.

M. K. Suh, M. Rofouei, A. Nahapetian, W.J. Kaiser, M. Sarrafzadeh (2009) Optimizing Interval Training Protocols Using Data Mining Decision Trees, IEEE, Berkeley: CA, USA.

A. Morrison, A. Davies, G. Brecevic, I. Sem, T. Boykett, R. Brecevic, Designing performativity for mixed reality installations, FORMakademisk, 3 (2010) 123-144.

A. Fiskerstrand, K.S. Seiler, Training and performance characteristics among Norwegian international rowers 1970-2001, Scandinavian Journal of Medicine \& Science in Sports, 14 (2004) 303-310. 
6. J. Esteve-Lanao, A.F. San Juan, C.P.

Earnest, C. Foster, A. Lucia, How do endurance runners actually train? Relationship with competition performance, Medicine \& Science in Sports \& 11. Exercise, 37 (2005) 496-504.

7. K.S. Seiler, G.Ø. Kjerland, Quantifying training intensity distribution in elite endurance athletes: is there evidence for an "optimal" distribution?, Scandinavian Journal of Medicine \& Science in 12 . Sports, 16 (2006) 49-56.

8. T.K. Tong, F.H. Fu, R. Eston, P.K. Chung, B. Quach, $\mathrm{K}$. Lu, Chronic and Acute Inspiratory Muscle Loading Augment the Effect of a 6-Week Interval Program on 13. Tolerance of High-Intensity Intermittent Bouts of Running, Journal of Strength and Conditioning Research, 24 (2010) 3041-3048.

9. A. Scanlan, B. Dascombe, P. Reaburn, A comparison 14 of the activity demands of elite and sub-elite Australian men's basketball competition, Journal of Sports Science, 29 (2011) 1153-1160.

10. P.B. Laursen, D.G. Jenkins, The scientific basis for high-intensity interval training: optimising training programmes

and maximizing performance in highly trained endurance athletes, Sports Medicine, 32 (2002) 53-73.

11. K.R. Barnes, W.G. Hopkins, M.R. McGuigan, A.E. Kilding, Effects of Different Uphill Interval-Training Programs on Running Economy and Performance, International Journal of Sports Physiology and Performance, 8 (2013) 639-647.

2. A.E. Kilding, M. Wood, G. Sequira, D.L. Bonetti, Effect of hyperoxic supplemented interval training on endurance performance in trained cyclists, International Journal of Sports Medicine, 33 (2012) 359-363.

13. G. Dupont, K. Akakpo, S. Berthoin, The effect of inseason, high-intensity interval training in soccer players, Journal of Strength and Conditioning Research, 18 (2004) 584-589.

14. M. Rakobowchuk, E. Harris, A. Taylor,

R.M. Cubbon, K.M. Birch, Moderate and heavy metabolic stress interval training improve arterial stiffness and heart rate dynamics in humans, European Journal of Applied Physiology, 113 (2013) 839-849.

Table1. Descriptive statistics and T-test result

\begin{tabular}{|c|c|c|c|c|c|c|c|c|}
\hline NO & Distance & Tests & Mean & $\begin{array}{c}\text { Mean } \\
\text { difference }\end{array}$ & Std & Df & t-value & p-value \\
\hline \multirow{2}{*}{1} & \multirow{2}{*}{$800 \mathrm{~m}$} & Pre & 2:09:40 & \multirow{2}{*}{ 00:01:09 } & 0:09:20 & \multirow{2}{*}{4} & \multirow{2}{*}{0.00} & \multirow{2}{*}{0.000} \\
\hline & & Post & 2:08:31 & & 0:05:46 & & & \\
\hline \multirow{2}{*}{2} & \multirow{2}{*}{$1500 \mathrm{~m}$} & Pre & 4:09:15 & \multirow[t]{2}{*}{ 00:04:06 } & $0: 24: 24$ & \multirow{2}{*}{6} & \multirow{2}{*}{3.39} & \multirow{2}{*}{0.015} \\
\hline & & Post & 4:05:09 & & $0: 25: 15$ & & & \\
\hline \multirow{3}{*}{3} & \multirow{2}{*}{$3 \mathrm{Km}$} & Pre & $8: 58: 43$ & \multirow{2}{*}{ 00:02:84 } & $0: 48: 52$ & \multirow{2}{*}{5} & \multirow{2}{*}{0.69} & \multirow{2}{*}{0.000} \\
\hline & & Post & 9:01:27 & & $0: 55: 56$ & & & \\
\hline & & Pre & 15:57:01 & & 1:21:22 & & & \\
\hline
\end{tabular}

Appendix

\begin{tabular}{|c|c|c|c|c|c|c|c|c|}
\hline $\mathbf{4}$ & $5 \mathrm{Km}$ & Post & $15: 56: 05$ & $00: 00: 56$ & $1: 31: 58$ & 7 & 0.01 & 0.000 \\
\hline \multirow{2}{*}{5} & \multirow{2}{*}{$10 \mathrm{Km}$} & Pre & $32: 08: 55$ & \multirow{2}{*}{$00: 52: 18$} & $3: 05: 18$ & \multirow{2}{*}{3} & \multirow{2}{*}{4.82} & 0.017 \\
\cline { 3 - 4 } & & Post & $31: 56: 37$ & & $3: 04: 10$ & & \\
\hline
\end{tabular}

$\mathbf{P}<\mathbf{0 . 0 5}$ 